\title{
Designing Stable and High-Current Density Rotor Windings of Superconducting Generator
}

\author{
Osami Tsukamoto and Mitsulo Furuse \\ Faculty of Engineering, Y'okohama National University, Yokohama, 240-8501 Japan \\ Tomoaki Takao \\ Faculty of Science and Technology, Sophia University, Tokyo, 102-8554 Japan \\ Masio Morita and Susumu Maeda \\ Mitsubishi Electric Corporation, Amagasisiki, 661-8661 Jilpann \\ Toshiki Hirao \\ Engineering Research Association for Superconductive Generation Equipment (Super-GM), Osaka, 530-0047 Japan
}

\begin{abstract}
A method to statistically predict quench training characteristics of a rotor windings of a superconducting generator is presented. In the method, energy of a disturbance due to a conductor motion and a quench current are statistically estimated. We apply this method to a rotor of the $70 \mathrm{MW}$ class Superconducting generator being developed in the Super-GM project and study the dependence of the stability on parameters of the rotor conductors, such as amount of copper stabilizer, accuracy of conductor dimensions and operating current density. To predict the' stability, compliance of the rotor winding pack is a key parameter and estimated by a finite element method. In the study, it is shown that there is an optimum value of ratio of copper to superconductor to maximize the current density of the winding pack keeping necessary stability. Based on the study, a designing method for stable and high current density rotor winding is discussed.
\end{abstract}

\section{INTRODUCTION}

One of key issues in designing a superconducting generator is how to design high current density rotor winding whose quench current surely exceed designed operation current. However, a method to quantitatively estimate a quench current of the winding has not been developed. This paper is related to this issue. MQE (Minimum Quench Energy) is a wellknown measure of the stability of superconductors but can not be a proper measure of the stability of the superconducting windings without knowledge on the size of disturbance in the windings. Therefore, it is important to know quanlitatively the size of the disturbance energy to estimate the stability.

Previously, we developed a theory to statistically predict the size of the disturbance caused by a conductor motion [1]. In the theory, parameters such as mechanical properties and amount of copper stabilizer of the conductor and configuration of winding pack are taken into consideration together with the MQE. We apply this theory to $70 \mathrm{MW}$ class superconducting generator under development in the Super-GM project. A key

Manuscript received September 14, 1998.

This work has been perfomed as a part of "R\&D on Superconducting Technology for Electric Power Apparaluses" under the New Sunshine Program of Agency of Industrial Science and Technology, MITI, being consigned by NEDO. parameter of the winding pack to predict the stability is its compliance which depends on the amount of copper stabilizer of the conductor, the property of the spacer and the configuration of the winding pack. We estimated the compliance of the winding pack by the finite element method. In the paper, the validity of the theory is shown by comparing the theoretical analysis and the test results. Based on the theory, possible methods to increase the current density of the rotor winding pack keeping the necessary stability and a design method of the stability is also discussed.

\section{PRINCIPLE TO STATISTICALLY ESTIMATE TRAINING CHARACTERISTICS}

\section{A Disturbance Energy and Maximum. Movable Length}

The rotor windings are usually wound of cable conductors composed of superconducting composite strands and we consider that a quench is most probably triggered by a motion of a strand of the conductors. The strands in the windings ave mainly supported by frictional forces. The strands inevitably have irregularities in their dimensions, hence, the frictional supporting forces of the strands fluctuate because contact lorces between the strands fluctuate along the strands even if uniform clamping force is applied to the windings. An abrupt strand motion occurs at a poorly supported part where the electromagnetic force to the strands exceeds the frictional supporting force, and, a quench occurs when the energy released by the strand motion at the poorly supported part is larger than the $\mathrm{MQE}$ of the strand. Energy released by the strand motion $E_{d}$ is delcrmined by the length of the moving part of the strand, that is, that of the poorly supported part,

$$
E_{d}=\frac{(B I)^{2}(1 / 2)^{5}}{45 E I_{d}}
$$

where $l$ is the length of the moving part of the strand, $B$ is the magnetic field component perpendicular to the direction of the strand motion, $I$ is the strand current, $E$ is Young's modulus of the strand, and $I_{d}$ is the geometrical moment of inemtia of the strand, $l_{d}=\pi r^{4} / 64$ for a round strand of the radius $r$. Knowing the relation between $E_{d}$ and $l$, we can define the maxinum movable length (MML), the maximum lengh of at part of the strand which can move without a quench. The MML is obtained from (1) by putting $E_{\text {r }}=M Q E$ and given by 


$$
M M L=\left\{\frac{1440(M Q E) E I_{d}}{(B I)^{2}}\right\}^{1 / 5}:
$$

\section{B. Prediction of Training Characteristics}

Assuming that the random irregularity of strand dimension follows the Gaussian process, we can calculate the expected number of poorly supported parts whose lengths exceed the MML and this number is the expected number of quenches of the windings. The expected number of quenches depends on the strand current because the MQE, MML and forces to the strand depend on the strand current. We can consider that the first quench occurs at the current when the expected number of quenches becomes 1 and that the next quench follows every time at the current where the expected number of quenches increases by one. Thus training characteristics of the windings can be predicted.

\section{II. CASE STUdY}

By applying the method mention above, we estimate the training characteristics of the rotor developed by Mitsubishi Electric Co. for the $70 \mathrm{MW}$ class superconducting generator as a part of the Super-GM projects.

\section{A. Configurations of Rotor Winding and Conductor}

The cross-sectional view of the rotor is shown in Fig. 1 and the specifications of the rotor are listed in Table I. The rotor windings are placed in the slots No.1 5. A cross-sectional view of the winding pack in the slot is illustrated in Fig. 2. Details of the rotor configuration are given in [2]. The crosssection of the conductor made of 9 strands is illustrated in Fig. 3 and the specifications of the conductor and strand are listed in Table II. The winding packs are surrounded by insulation spacers as shown in Fig. 2 and the compressive forces are applied to the packs and conductors by the clamping forces to the top insulation spacers. The rotor shaft with the windings in the slots was fitted in the rotor vessel by shrink-fit and the clamping forces were applied to the top insulation spacer by the vessel.

\section{B. Forces to Conductor and Strand}

When the rotor rotates and the windings are excited, the centrifugal forces and the electromagnetic forces are applied to the conductors in addition to the compressive force from the upper insulation spacer. The electromagnetic forces have two components of circumferential and radial directions of the rotor. The circumferential component of the electromagnetic force causes the strand motions. The distributions of the magnetic field and the electromatgnetic forces to the conductors in the slots are estimated based on the analysis using the finite element method. The distribution of total forces to the conductors in the slots can be calculated by knowing the compliance of the winding pack in the radial direction.

\section{Compliance of Rotor Winding}

The strand of the rotor winding contain CuNi to reduce $A C$ losses. Therefore, stress-strain characteristics of the rotor

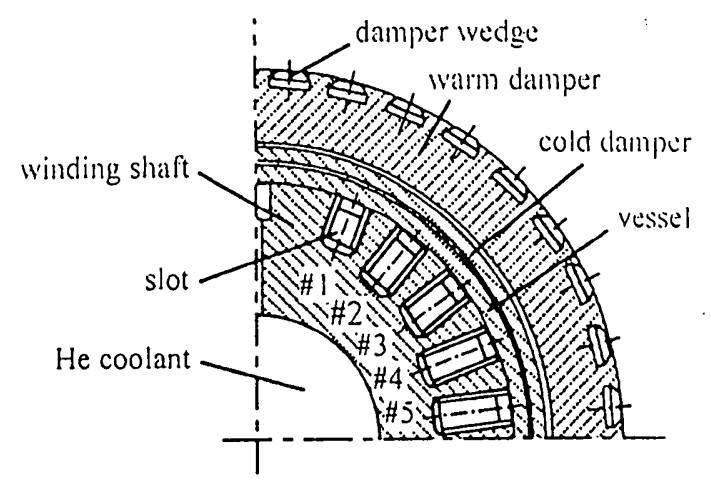

Fig. 1. Cross-section of rolor (perpendicular to center axis, quarter of the rolor).

TABLE I

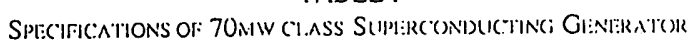

\begin{tabular}{ll}
\hline Rotor & \\
Number of polc & 2 \\
Rotaling velocity & $3600 \mathrm{rpm}$ \\
Dianeter & $890 \mathrm{~mm}$ \\
Bearing span & $4900 \mathrm{~mm}$ \\
Winding & \\
No. of coils & $10(5$ coils/pole) \\
Magnetic licld (maximum) & $4.5 \%$ (at $3000 \mathrm{~A})$ \\
Conductor & 9 stlind compacted cablc \\
\hline
\end{tabular}

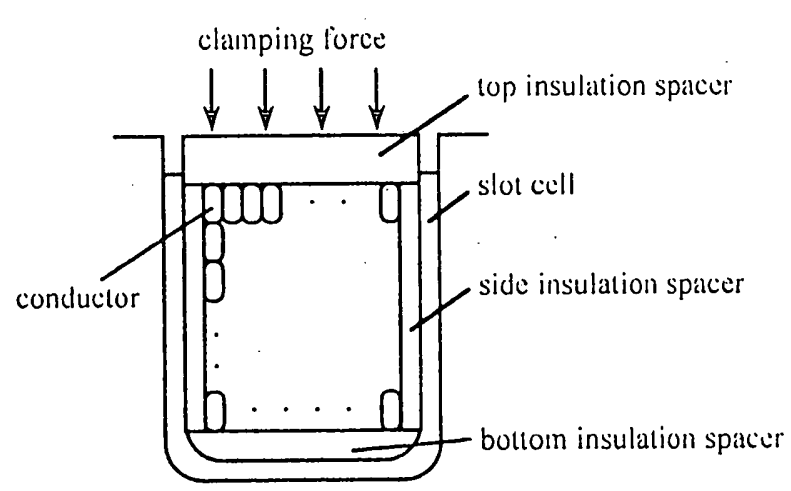

Fig. 2. Cross-sectional view of the winding pack in the slot.

winding pack in the radial direction were calculated for different values of ratio of copper stabilizer and $\mathrm{CuNi}$ to NbTi by the finite element method. We call this ratio nonSc/Sc after this. In the calculation, we changed nonSc/Sc by changing the amount of outer layer copper stabilizer while fixing the amount of $\mathrm{CuNi}$. The compliances were calculated for the strand of same configuration shown in Fig. 3 except nonSc/Sc. In the calculation, stress-strain datit of $\mathrm{Cu}, \mathrm{NbTi}$ and $\mathrm{CuNi}$ were used and it was assumed that the core part of the strand including NbTi filamentary region stayed in the elastic region and that stabilizing copper region went in the plastic region. The stress-strain characteristics of the insulating kapton tape given in the reference [3] were also taken into consideration. The results are shown in Fig. 4 . The compressive forces to the strands fluctuate around the clamping force. Therefore, the compliance of the winding pack is detemined from the slope of the stress-strain curve at the stress determined by the clamping force. Obviously from Fig. 4, the compliance increases as the nonSc/Sc increase, because the anount of soft copper stabilizer increases. 


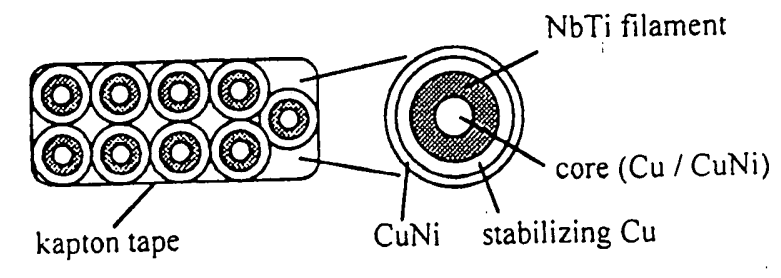

Fig. 3. Cross-sections of rotor conductor and strand.

TABLE II

SPECIFCATIONS OF CONDLCTOR AND STRAND

\begin{tabular}{ll}
\hline Conductor (compacted strand cable) & \\
Size in cross section & $2.9 \mathrm{~mm} \times 7.4 \mathrm{~mm}$ \\
No. of strands & 9 strands \\
Critical current & $5100 \mathrm{~A}(7.6 \mathrm{~T})$ at $4.2 \mathrm{~K}$ \\
& $4900 \mathrm{~A}(7.3 \mathrm{~T})$ at $4.6 \mathrm{~K}$ \\
Strand & $2: 0.7: 1$ \\
Cu: CuNi : NbTi & $1.6 \mathrm{~mm}$ \\
Diameter & $130 \mathrm{GPa}$ \\
Young's modulus & $3.2 \times 10^{-13} \mathrm{~m}^{4}$ \\
\hline
\end{tabular}

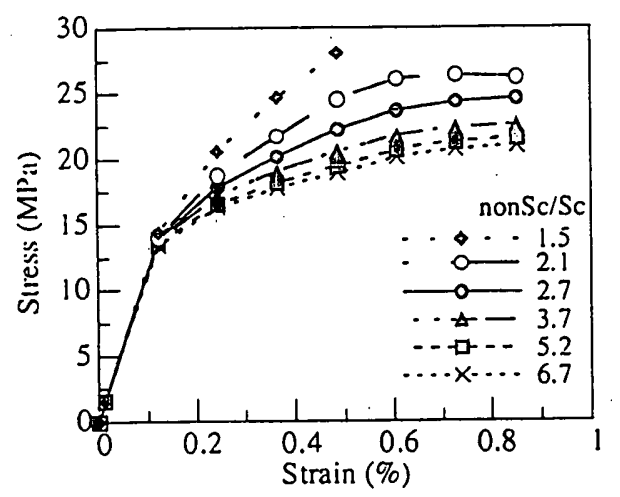

Fig. 4. Stress-strain curves of winding pack of different non Sc/Sc conductors.

\section{Calculation of $M Q E$ and $M M L$}

We assume that a quench of the winding is initiated by a quench caused by a strand motion in the conductor, therefore, $M Q E$ of the single strand is calculated. Details of calculation of MQE are described in [4]. We assumed a point disturbance of $0.5 \mathrm{msec}$ duration [5] and $2 \mathrm{~mm}$ spatial length to calculate MQE. The spatial length of the disturbance was determined by the observation of the conductor configuration. MQE's were calculated in the static and rotating conditions. In the calculation, the transient heat transfer of liquid helium was taken into account in the static condition. In the rotating condition. data of the steady heat transfer of the supercritical helium in high centrifugal field [6] were used, because, according to our experiment. the steady state heat transfer was established in very short time [7].

\section{E. Calculation of Expected Number of Quenches and Training Characteristics}

We assume the irregularity in the dimension of the conductor follows the Gaussian process of standard deviation $\sigma$, then the dimension irregularity of the winding pack also follows the Gaussian process whose standard deviation is $\sqrt{17} \sigma$, where $n$ is the number of the layers of the winding pack. It can be considered the contact forces between the conductors also follows the Gaussian process whose standard deviation is $k \sqrt{n} \sigma$ where $k$ is the spring constant of the winding pack which can be deduced from the compliance of the winding pack calculated by the finite element method. Then, we can numerically simulate the distribution of the fluctuation of the contact force along the conductor using the method explained in [1]. We can assume that the contact forces between conductors are equal to that between the strands of the conductor. Thus, by multiplying the contact forces by the friction coefficient $\mu$ between the strands, we can estimate the distribution of the supporting force along the strand and the number of the poorly supported parts whose lengths exceed the MML for a given conductor current. This number is the expected number of quenches till the conductor current reaches the given value.

\section{F. Comparison of Predicted Training Characteristics with Experimental Result}

The quench test of the rotor winding which is case-studied here was performed in the static condition. nonSc/Sc of the strand is 2.7. The validity of the prediction method can be checked by comparing the predicted training characteristics with the experimental result. Using the parameters listed in Table III, the accumulation of the expected number of the quenches until the conductor current reached $I$ was calculated for the non $\mathrm{Sc} / \mathrm{Sc}=2.7$ for the static conditions. $\sigma=33 \mu \mathrm{m}$ was determined based on the specified dimension allowance of the conductors and $\mu=1.0$ was determined based on experimental value of the copper vs. copper friction [8]. Fig. 5 shows the estimated training characteristics. As seen in the figure, the first quench current is $4.4 \mathrm{kA}$ for the static condition. It is also seen that the first quench is followed by many successive quenches.

Result of the quench test in the static condition is shown in Fig. 6. The current at the first quench is $4.3 \mathrm{kA}$ and many quenches follows as is seen in the calculation result in Fig. 5: Based on this comparison, we can assume that the method to predict the training characteristics is valid.

\section{G. Dependence of Stability on Amount of Copper Stabilizer}

As nonSc/Sc increases, the spring constant $k$ of the winding pack decreases and the standard deviation of the contact forces between the strands decreases. Therefore, the number of the poorly supported parts whose lengths exceed the MML decreases and the conductor current reaches its critical value with smaller number of the training quenches. However, high nonSc/Sc sacrifices the current density. On the other hand, low nonSc/Sc strand causes large winding pack spring constant and severe training effect.

From the standpoint of designing the rotor winding, it is important that the first quench current is high. Obviously from the discussion above, there is an optimum value of nonSc/Sc to make the first quench current maximum for given clamping force to the winding pack and $\sigma$. Fig. 7 shows the dependence of the first quench current on the nonSc/Sc. The dependence is estimated for a conductor of same dimensions shown in Fig. 3 for different values of $\sigma$ by the method described above. The parameters for the calculation are same as in Table III except $\sigma$. The critical current of the conductor $I_{\text {c }}$ on the load line, depending on the nonSc/Sc. is also shown in 
TABLE III

Paramittirs on Calculation of Expected Number of QuenCh

\begin{tabular}{ll}
\hline$\sigma$ & $3.3 \mu \mathrm{m}$ \\
$\mu$ & 1.0 \\
Spring constant of winding pack & $5.5 \times 10^{7}[\mathrm{~N} / \mathrm{m} / \mathrm{strand}] / \mathrm{m}$ (static condition) \\
$\quad$ (nonSc/Sc $=2.7)$ & $3.7 \times 10^{7}[\mathrm{~N} / \mathrm{m} / \mathrm{strand}] / \mathrm{m}$ (rotiting condition) \\
Clamping forcc to the windings & $5.2 \times 10^{4} \mathrm{~N} / \mathrm{m} /$ conductor (static condition) \\
& $6.2 \times 10^{4} \mathrm{~N} / \mathrm{m} /$ conductor (rotating condition) \\
\hline
\end{tabular}

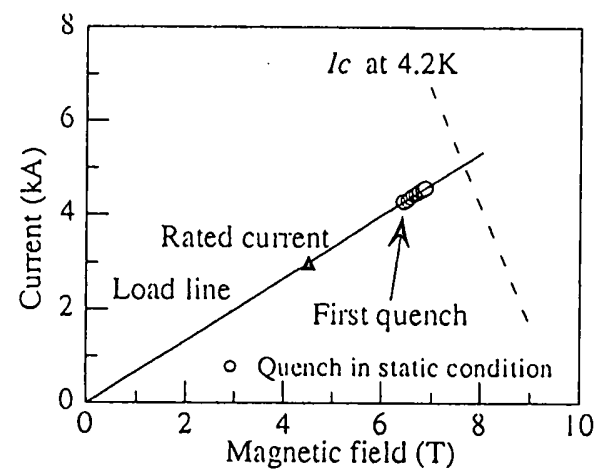

Fig. 5. Prediction of training quenches of the winding in the static condition.

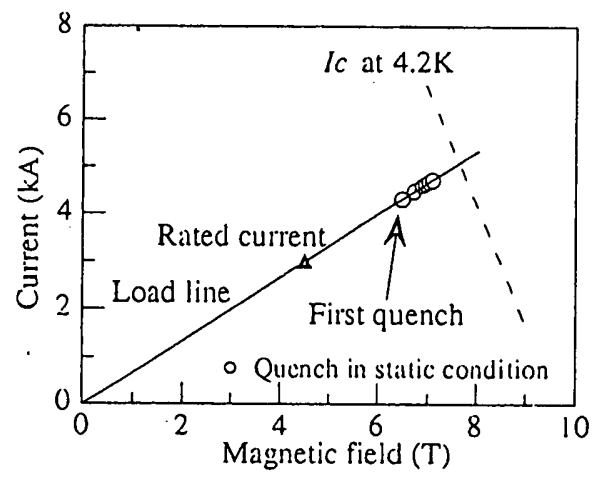

Fig. 6. Result of training quench test.

Fig. 7. For the case of $\sigma=33 \mu \mathrm{m}$, the first quench current becomes maximum at nonSc/Sc=3.7 and is $4.6 \mathrm{kA}$ which is $I_{c}$ at the point. For nonSc/Sc higher than 3.7, the conductor current reaches $I_{c}$ without quench but current capacity of the conductor becomes lower.

To obtain higher first quench current, it is necessary to decrease $\sigma$ for a conductor of lower nonSc/Sc to reach the maximum first quench current. The maximum values are 4.9kA for $\sigma=25 \mu \mathrm{m}$ at nonSc $/ \mathrm{Sc}=2.7$ and $5.1 \mathrm{kA}$ for $\sigma=20 \mu \mathrm{m}$ at nonSc/Sc $=2.1$. The first quench can be also increased by increase of the clamping force to the winding but too high clamping force deteriorates the conductor performance damaging the conductor.

\section{CONCluding Remarks - Designing Stable and High CURRENT DENSITY WINDING}

Summarizing the study above, the following conclusions are obtained.

1. Quantitative estimation of the first quench and design of the stability of the winding are possible by the method described in the paper.

2. For a given value of $\sigma$ and clamping force to the

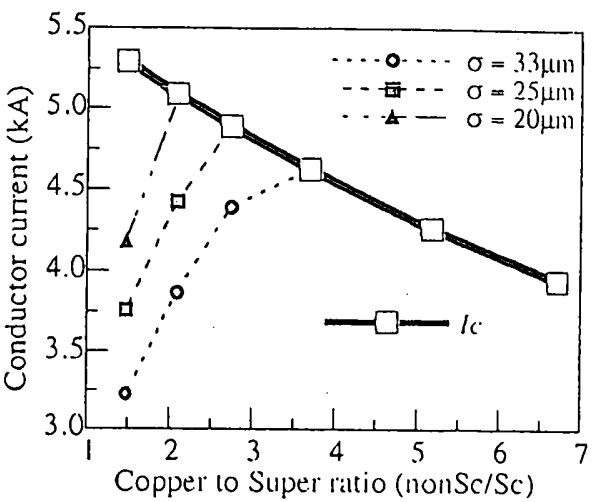

Fig. 7. Dependence of first quench current on nonSe/Sc for different valuess of $\sigma$.

winding, there is an optimum value of nonSc/Sc to make the first quench current maxinum. Low value of nonSc/Sc, that is, high $l_{\text {c }}$ does not necessarily make the current density of the winding high. Increase of nonSc/Sc contributes to the reduction of the conductor cost by reducing the amount of $\mathrm{NbTi}$.

3. Data of the compliance of the winding pack are key of our stability estimation method, we need more knowledge and data on the compliance of the wincling.

\section{ACKNOWLEDGMENT}

The authors are very grateful to Prof. M. Shiratori and Dr. J. Gotoh of Faculty of Engineering, Yokohama National University to assist us to calculate the compliance of the winding using the finite element method.

\section{REFERENCES}

[1] T. Takato, K. Iwasaki and O. Tsukamolo, "Statistical Estimation of Disturbance Energy duc to Conductor Motion in Rotor Windings of Superconducting Gencrator," Il:E: Trems. (m Appl. Super., vol. 5. No. 2 pp. $361-364,1995$

[2] T. Ohara, H. Fukuda, T. Ogawa, K. Shiobara, M. Ohi, A. Ueda, K. Ioh and H. Taniguchi, "Development of $70 \mathrm{MW}$ class superconducting generators," IEEE Trans. on Magn., vol.27, No.2, pp2232-2239, 1991.

[3] R. P. Reed, R. E. Schramm and A. F. Clard, "Mechanical, themal and electrical properties of selected polymers," Cryogenics, pp. 66-82, 1973.

14] N. Amemiya, T. Takao and O. Tsukamolo, "Disturbance characteristics and stability of high current density superconducting wircs," Fussion Engineering Design, vol. 20, pp. 339-344, 1993.

15] O. Tsukamoto, "Disturbance due to conductor motion and stability of large-scale conductors," Fusion Engine ering Desisn, vol. 20, pp. 327-332, 1993.

[6] R. Nakiajima, K. Salo, K. Miyaike, M. Kumagai and Y. Kobalyashi, "Liquid and supercritical helium heat transfer of horizontal upward facing surfaces under high centrilugal acceleration fields," HTD-Vol. 229. Heat transfer in superconducting equipment, G00721, pp. 39-44, 1992.

[7] O. Tsukamolo, M. Furuse, T. Takan, N. Tamada, S. Fuchino, 1. Ishii and N.Higuchi, "Transient heall transfer characteristics of liquid helium in centrifugal acceleration lield," presented al CEC/ICMC97.

18] T. Takalo, D. Yoshino, T. Kubosaka, N. Momma, I. Nilla, A. I wabuchi, F. Odajima, "Influence of Surface Roughness and Precooling by Liquid Nitrogen on Frictional Coeflicients at Liquid Helium Temperallure." presented at 17th ICEC, 1998 